\title{
The Poisson-weighted Sujatha Distribution with Properties and Applications
}

\author{
Rama Shanker ${ }^{1}$, Kamlesh Kumar Shukla ${ }^{2^{*}}$ \\ ${ }^{1}$ Department of Statistics, College of Science, Eritrea Institute of Technology, Asmara, Eritrea \\ ${ }^{2}$ Department of Statistics, College of Science, Eritrea Institute of Technology, Asmara, Eritrea \\ *Corresponding Author: kkshukla22@gmail.com
}

Available online at: www.isroset.org

Received: 02/Sept/2018, Accepted 11/Oct/2018, Online 31/Oct/2018

\begin{abstract}
The Poisson distribution is an important discrete distribution for modeling count data having equi-dispersion. In this paper, a new discrete distribution for modeling count data having over-dispersion, namely, the Poisson-weighted Sujatha distribution which includes Poisson-Sujatha distribution has been proposed by compounding Poisson distribution with a twoparameter weighted Sujatha distribution. Its statistical properties including moments, coefficient of variation, skewness, kurtosis, index of dispersion, unimodality and increasing hazard rate have been discussed. Maximum likelihood estimation has been explained for estimating its parameters. Applications of the distribution have been discussed with some count datasets and its goodness of fit has been compared with other discrete distributions having over-dispersion.
\end{abstract}

Keywords: Poisson- Sujatha distribution, Weighted- Sujatha distribution, Compounding, Moments, Skewness, Kurtosis, Maximum likelihood estimation, Applications.

2010 Mathematics Subject Classification: Primary 62 E 05; Secondary 62 E 99

\section{INTRODUCTION}

The statistical analysis and modeling of count data are crucial in almost all fields of knowledge including biological science, insurance, medical science, finance, are some among others. Count data are generated from different phenomena such as the number of insurance claimants in insurance, number of yeast cells in biological science, number of chromosomes in genetics, etc. It has been observed that, in general, count data follows under-dispersion (variance $<$ mean), equi-dispersion $($ variance $=$ mean) or over-dispersion (variance > mean). The over-dispersion of count data have been addressed using mixed Poisson distributions by different researchers including Raghavachari et al [1], Karlis and Xekalaki [2], Panjeer [3], are some among others. Mixed Poisson distributions arise when the parameter of the Poisson distribution is a random variable having some specified distributions. The distribution of the parameter of the Poisson distribution is known as mixing distribution. It has been observed that the general characteristics of the mixed Poisson distribution follow some characteristics of its mixing distributions. The field of distribution theory is flooded with mixed Poisson distributions based on a number of proper mixing distributions.

The classical negative binomial distribution (NBD) derived by Greenwood and Yule [4] is the mixed Poisson distribution where the parameter of the Poisson random variable is distributed as a gamma random variable. The NBD has been used to model over-dispersed count data. However, the NBD may not be appropriate for some over-dispersed count data due to its theoretical or applied point of view. Other mixed Poisson distributions arise from a choice of alternative mixing distributions. For example, the Poisson-Lindley distribution, introduced by Sankaran [5], is a Poisson mixture of Lindley [6] distribution. The Poisson-Akash distribution, introduced by Shanker [7], is a Poisson mixture of Akash distribution proposed by Shanker [8]. The generalized Poisson-Lindley distribution, introduced by Mahmoudi and Zakerzadeh [9], is a mixed Poisson distribution where the mixing distribution is the generalized Lindley distribution proposed by Zakerzadeh and Dolati [10]. Other mixed distributions are the Poisson-weighted exponential distribution (P-WED) introduced by Zamani et al [11] and the negative binomial-Lindley distribution (NB-LD) introduced by Zamani and Ismail [12] where the mixing distributions were weighted exponential distribution and Lindley[6] distribution, respectively. The Poisson-weighted Lindley distribution (P- 
WLD) introduced by Abd EL-Monsef and Sohsah [13] is a Poisson mixture of weighted Lindley distribution proposed by Ghitany et al [14].

It has been observed by Karlis and Xekalaki [2] that there are naturally situations where a good fit is not obtainable with a particular mixed Poisson distribution in case of over-dispersed count data. This shows that there is a requirement for new mixed Poisson distribution which gives better fit as compared with the existing mixed Poisson distributions.

Shanker [15] introduced Poisson-Sujatha distribution (PSD) defined by its probability mass function (pmf)

$$
P_{1}(x ; \theta)=\frac{\theta^{3}}{\theta^{2}+\theta+2} \cdot \frac{x^{2}+(\theta+4) x+\left(\theta^{2}+3 \theta+4\right)}{(\theta+1)^{x+3}} ; x=0,1,2, \ldots, \theta>0
$$

Statistical properties including shapes of pmf for varying values of parameter, coefficient of variation, skewness, kurtosis, index of dispersion, unimodality, increasing hazard rate have been discussed by Shanker [15]. Further, the estimation of parameter using both the method of moment and the method of maximum likelihood along with applications of PSD has been discussed by Shanker [15]. Note that PSD arises from the Poisson distribution where the mixing distribution is the Sujatha distribution introduced by Shanker [16] having probability density function (pdf)

$$
f_{1}(\lambda, \theta)=\frac{\theta^{3}}{\theta^{2}+\theta+2}\left(1+\lambda+\lambda^{2}\right) e^{-\theta \lambda} ; \lambda>0, \theta>0
$$

Shanker [16] has discussed several important statistical and mathematical properties of Sujatha distribution including moments and moments based measures, hazard rate function, mean residual life function, mean deviations from the mean and the median, stochastic ordering, Bonferroni and Lorenz curves and stress-strength reliability, are some among others. Further, Shanker [16] has discussed the method of moment and the method of maximum likelihood estimation for estimating the parameter of Sujatha distribution. The applications and goodness of fit of Sujatha distribution have also been discussed by Shanker[16] with some real lifetime datasets from biomedical sciences and engineering. Shanker [15] has shown that PSD is a better model than the Poisson-Lindley distribution (PLD) introduced by Sankaran [5] having pmf

$$
P_{2}(x ; \theta)=\frac{\theta^{2}(x+\theta+2)}{(\theta+1)^{x+3}} ; x=0,1,2, \ldots, \theta>0
$$

Note that PLD is a Poisson mixture of Lindley distribution introduced by Lindley [6] having pdf

$$
f_{2}(x, \theta)=\frac{\theta^{2}}{\theta+1}(1+x) e^{-\theta x} ; x>0, \theta>0
$$

Recently Shanker and Shukla (2018)[17] have introduced a two-parameter weighted Sujatha distribution (WSD) defined by its pdf

$$
f_{3}(x ; \theta, \alpha)=\frac{\theta^{\alpha+2}}{\theta^{2}+\alpha \theta+\alpha(\alpha+1)} \frac{x^{\alpha-1}}{\Gamma(\alpha)}\left(1+x+x^{2}\right) e^{-\theta x} ; x>0, \theta>0, \alpha>0
$$

where $\Gamma(\alpha)=\int_{0}^{\infty} e^{-y} y^{\alpha-1} d y ; \alpha>0 \quad$ is the complete gamma function. It can be seen that at $\alpha=1$, it reduces to the one parameter Sujatha distribution (1.2). This distribution has been found to be a better model than the one parameter Sujatha distribution for analyzing and modeling lifetime data from engineering. Shanker and Shukla [17] have derived and discussed various statistical properties of WSD including moments and moments based measures, hazard rate function, mean residual life function, stochastic ordering, some among others. Further, Shanker and Shukla [17] have discussed the method of maximum likelihood for estimating the parameter of WSD. 
Ghitany et al [14] introduced a two-parameter weighted Lindley distribution (WLD) having parameters $\theta$ and $\alpha$ and defined by its pdf

$$
f_{4}(x ; \theta, \alpha)=\frac{\theta^{\alpha+1}}{(\theta+\alpha)} \frac{x^{\alpha-1}}{\Gamma(\alpha)}(1+x) e^{-\theta x} ; x>0, \theta>0, \alpha>0
$$

Its structural properties including moments, hazard rate function, mean residual life function, estimation of parameters and applications for modeling survival time data has been discussed by Ghitany et al [14]. The corresponding cumulative distribution function (cdf) of WLD (1.5) is given by

$$
F(x ; \theta, \alpha)=1-\frac{(\theta+\alpha) \Gamma(\alpha, \theta x)+(\theta x)^{\alpha} e^{-\theta x}}{(\theta+\alpha) \Gamma(\alpha)} ; x>0, \theta>0, \alpha>0
$$

where

$$
\Gamma(\alpha, z)=\int_{z}^{\infty} e^{-y} y^{\alpha-1} d y ; \alpha>0, z \geq 0
$$

is the upper incomplete gamma function. It can be easily shown that at $\alpha=1$, WLD reduces to Lindley [6] distribution. Shanker et al [18] discussed various moments based properties including coefficient of variation, coefficient of skewness, coefficient of kurtosis and index of dispersion of WLD and its applications to model lifetime data from biomedical sciences and engineering. Shanker et al [19] have proposed a three-parameter weighted Lindley distribution (TPWLD) which includes a twoparameter weighted Lindley distribution and one parameter Lindley distribution as particular cases and discussed its various structural properties, estimation of parameters and applications for modeling lifetime data from engineering and biomedical sciences.

Assuming that the parameter $\lambda$ of the Poisson distribution follows WLD (1.5), Abd El-Monsef and Sohsah[13] proposed Poisson- weighted Lindley distribution (P-WLD) defined by its pmf

$$
P_{3}(x ; \theta, \alpha)=\frac{\Gamma(x+\alpha)}{\Gamma(x+1) \Gamma(\alpha)} \frac{\theta^{\alpha+1}}{(\theta+\alpha)} \frac{x+\theta+\alpha+1}{(\theta+1)^{x+\alpha+1}} ; x=0,1,2, \ldots, \theta>0, \alpha>0
$$

It can be easily verified that PLD (1.3) is a particular case of P-WLD for $\alpha=1$.

Since WSD is a better model than Sujatha, Lindley and weighted Lindley distributions for modeling lifetime data and PSD is a better model than PLD for modeling count data, it is expected and hoped that a Poisson mixture of WSD will provide a better model than Poisson, PLD, PSD and P-WLD for modeling count data. Keeping these points in mind, a Poisson mixture of WSD has been introduced and studied. Its various statistical properties based on moments, increasing hazard rate and estimation of parameters using the method of maximum likelihood along with applications have been discussed.

\section{THE POISSON-WEIGHTED SUJATHA DISTRIBUTION}

Assuming that the parameter $\lambda$ of the Poisson distribution follows WSD (1.5), the Poisson mixture of WSD can be obtained as

$$
P_{4}(x ; \theta, \alpha)=\int_{0}^{\infty} \frac{e^{-\lambda} \lambda^{x}}{\Gamma(x+1)} \frac{\theta^{\alpha+2}}{\theta^{2}+\alpha \theta+\alpha(\alpha+1)} \frac{\lambda^{\alpha-1}}{\Gamma(\alpha)}\left(1+\lambda+\lambda^{2}\right) e^{-\theta \lambda} d \lambda
$$




$$
\begin{aligned}
& =\frac{\theta^{\alpha+2}}{\left[\theta^{2}+\alpha \theta+\alpha(\alpha+1)\right] \Gamma(\alpha) \Gamma(x+1)}\left[\int_{0}^{\infty} e^{-(\theta+1) \lambda} \lambda^{x+\alpha-1} d \lambda+\int_{0}^{\infty} e^{-(\theta+1) \lambda} \lambda^{x+\alpha+1-1} d \lambda\right] \\
& =\frac{\theta^{\alpha+2}}{\left[\theta^{2}+\alpha \theta+\alpha(\alpha+1)\right] \Gamma(\alpha) \Gamma(x+1)}\left[\frac{\Gamma(x+\alpha)}{(\theta+1)^{x+\alpha}}+\frac{\Gamma(x+\alpha+1)}{(\theta+1)^{x+\alpha+1}}+\frac{\Gamma(x+\alpha+2)}{(\theta+1)^{x+\alpha+2}}\right] \\
& =\frac{\theta^{\alpha+2}}{\Gamma(\alpha) \Gamma(x+1)} \frac{x^{2}+(\theta+2 \alpha+2) x+\left\{\theta^{2}+(\alpha+2) \theta+\left(\alpha^{2}+2 \alpha+1\right)\right\}}{(\theta+\alpha \theta+\alpha(\alpha+1)\}} \frac{(\theta+1)^{x+\alpha+2}}{; \theta^{2}+\alpha=0,1,2, \ldots, \theta>0, \alpha>0}
\end{aligned}
$$

We would call this pmf the Poisson - Weighted Sujatha distribution (P-WSD). It can be easily verified that PSD (1.1) is a particular case of P-WSD for $\alpha=1$. The natures of P-WSD for varying values of the parameters $\theta$ and $\alpha$ have been explained graphically in figure 1.
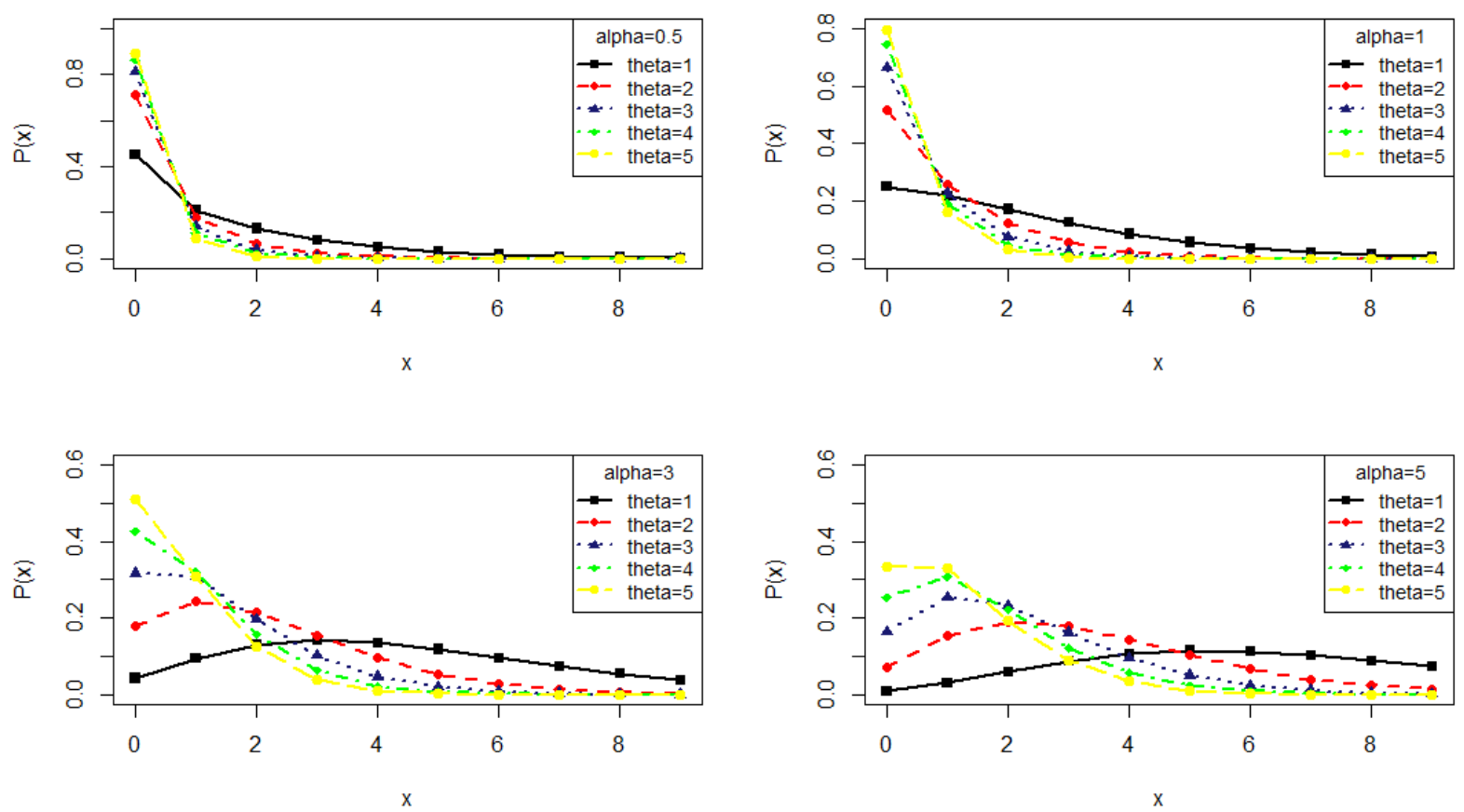

Fig. 1: Probability mass function plot of P-WSD for varying values of parameters $\theta$ and $\alpha$.

\section{STATISTICAL PROPERTIES OF P-WSD}

\section{A. Factorial Moments}

Using (2.1), the $r$ th factorial moment about origin, $\mu_{(r)}{ }^{\prime}$ of the P-WSD (2.2) can be obtained as

$$
\mu_{(r)}{ }^{\prime}=E\left[E\left(X^{(r)} \mid \lambda\right)\right], \text { where } X^{(r)}=X(X-1)(X-2) \ldots(X-r+1)
$$




$$
\begin{aligned}
& =\int_{0}^{\infty}\left[\sum_{x=1}^{\infty} x^{(r)} \frac{e^{-\lambda} \lambda^{x}}{\Gamma(x+1)}\right] \frac{\theta^{\alpha+2}}{\left\{\theta^{2}+\alpha \theta+\alpha(\alpha+1)\right\}} \frac{\lambda^{\alpha-1}}{\Gamma(\alpha)}\left(1+\lambda+\lambda^{2}\right) e^{-\theta \lambda} d \lambda \\
& =\frac{\theta^{\alpha+2}}{\left\{\theta^{2}+\alpha \theta+\alpha(\alpha+1)\right\} \Gamma(\alpha)} \int_{0}^{\infty}\left[\lambda^{r}\left\{\sum_{x=r}^{\infty} \frac{e^{-\lambda} \lambda^{x-r}}{(x-r) !}\right\}\right] \lambda^{\alpha-1}\left(1+\lambda+\lambda^{2}\right) e^{-\theta \lambda} d \lambda
\end{aligned}
$$

Taking $x-r=y$, we get

$$
\begin{aligned}
\mu_{(r)}^{\prime}{ }^{\prime} & =\frac{\theta^{\alpha+2}}{\left\{\theta^{2}+\alpha \theta+\alpha(\alpha+1)\right\} \Gamma(\alpha)} \int_{0}^{\infty}\left[\lambda^{r}\left\{\sum_{y=0}^{\infty} \frac{e^{-\lambda} \lambda^{y}}{y !}\right\}\right] \lambda^{\alpha-1}\left(1+\lambda+\lambda^{2}\right) e^{-\theta \lambda} d \lambda \\
& =\frac{\theta^{\alpha+2}}{\left\{\theta^{2}+\alpha \theta+\alpha(\alpha+1)\right\} \Gamma(\alpha)} \int_{0}^{\infty} \lambda^{\alpha+r-1}\left(1+\lambda+\lambda^{2}\right) e^{-\theta \lambda} d \lambda \\
& =\frac{\Gamma(\alpha+r)}{\Gamma(\alpha)} \frac{\theta^{2}+(\alpha+r) \theta+(\alpha+r)(\alpha+r+1)}{\theta^{r}\left\{\theta^{2}+\alpha \theta+\alpha(\alpha+1)\right\}} ; r=1,2,3, \ldots
\end{aligned}
$$

Taking $r=1,2,3$, and 4 in (3.1.1), the first four factorial moments about origin of P-WSD (2.2) can be obtained

$$
\begin{aligned}
& \mu_{(1)}^{\prime}=\frac{\alpha\left\{\theta^{2}+(\alpha+1) \theta+(\alpha+1)(\alpha+2)\right\}}{\theta\left\{\theta^{2}+\alpha \theta+\alpha(\alpha+1)\right\}} \\
& \mu_{(2)}^{\prime}=\frac{\alpha(\alpha+1)\left\{\theta^{2}+(\alpha+2) \theta+(\alpha+2)(\alpha+3)\right\}}{\theta^{2}\left\{\theta^{2}+\alpha \theta+\alpha(\alpha+1)\right\}} \\
& \mu_{(3)}^{\prime}=\frac{\alpha(\alpha+1)(\alpha+2)\left\{\theta^{2}+(\alpha+3) \theta+(\alpha+3)(\alpha+4)\right\}}{\theta^{3}\left\{\theta^{2}+\alpha \theta+\alpha(\alpha+1)\right\}} \\
& \mu_{(4)}^{\prime}=\frac{\alpha(\alpha+1)(\alpha+2)(\alpha+3)\left\{\theta^{2}+(\alpha+4) \theta+(\alpha+4)(\alpha+5)\right\}}{\theta^{4}\left\{\theta^{2}+\alpha \theta+\alpha(\alpha+1)\right\}} .
\end{aligned}
$$

\section{B.Raw Moments (Moments about Origin)}

Using the relationship between raw moments and the factorial moments about origin, the raw moments of P-WSD are obtained as

$$
\mu_{1}^{\prime}=\mu_{(1)}^{\prime}=\frac{\alpha\left\{\theta^{2}+(\alpha+1) \theta+(\alpha+1)(\alpha+2)\right\}}{\theta\left\{\theta^{2}+\alpha \theta+\alpha(\alpha+1)\right\}}
$$




$$
\begin{gathered}
\mu_{2}^{\prime}=\mu_{(2)}{ }^{\prime}+\mu_{(1)}{ }^{\prime}=\frac{\alpha\left\{\theta^{3}+2(\alpha+1) \theta^{2}+2\left(\alpha^{2}+3 \alpha+2\right) \theta+\left(\alpha^{3}+6 \alpha^{2}+11 \alpha+6\right)\right\}}{\theta^{2}\left\{\theta^{2}+\alpha \theta+\alpha(\alpha+1)\right\}} \\
\mu_{3}^{\prime}=\mu_{(3)}{ }^{\prime}+3 \mu_{(2)}{ }^{\prime}+\mu_{(1)}{ }^{\prime} \\
\mu_{4}^{\prime}=\mu_{(4)}{ }^{\prime}+6 \mu_{(3)}{ }^{\prime}+7 \mu_{(2)}{ }^{\prime}+\mu_{(1)}{ }^{\prime} \\
=\frac{\alpha\left\{\theta^{4}+4(\alpha+1) \theta^{3}+5\left(\alpha^{2}+3 \alpha+2\right) \theta^{2}+4\left(\alpha^{3}+6 \alpha^{2}+11 \alpha+6\right) \theta\right\}}{\theta^{3}\left\{\theta^{2}+\alpha \theta+\alpha(\alpha+1)\right\}} \\
\alpha\left\{\begin{array}{c}
\left.\theta^{5}+8(\alpha+1) \theta^{4}+14\left(\alpha^{2}+3 \alpha+2\right) \alpha^{3}+35 \alpha^{2}+50 \alpha+24\right) \\
+7\left(\alpha^{4}+10 \alpha^{3}+35 \alpha^{2}+50 \alpha+24\right) \theta+\left(\alpha^{5}+15 \alpha^{4}+85 \alpha^{3}+225 \alpha^{2}+274 \alpha+120\right)
\end{array}\right.
\end{gathered}
$$

\section{Central Moments (Moments about Mean)}

Now, using the relationship $\mu_{r}=E\left(Y-\mu_{1}^{\prime}\right)^{r}=\sum_{k=0}^{r}\left(\begin{array}{l}r \\ k\end{array}\right) \mu_{k}^{\prime}\left(-\mu_{1}^{\prime}\right)^{r-k}$ between central moments and the raw moments, the central moments of the P-WSD (2.2) can be obtained as

$$
\begin{aligned}
& \mu_{2}=\frac{\alpha\left\{\begin{array}{l}
\theta^{5}+2(\alpha+1) \theta^{4}+\left(3 \alpha^{2}+7 \alpha+4\right) \theta^{3}+14\left(2 \alpha^{3}+8 \alpha^{2}+12 \alpha+6\right) \theta^{2} \\
+\left(\alpha^{4}+6 \alpha^{3}+11 \alpha^{2}+6 \alpha\right) \theta+\left(\alpha^{4}+4 \alpha^{3}+5 \alpha^{2}+2 \alpha\right)
\end{array}\right\}}{\theta^{2}\left\{\theta^{2}+\alpha \theta+\alpha(\alpha+1)\right\}^{2}} \\
& \mu_{3}=\frac{\left\{\begin{array}{l}
\theta^{8}+(3 \alpha+4) \theta^{7}+\left(6 \alpha^{2}+16 \alpha+10\right) \theta^{6}+\left(7 \alpha^{3}+31 \alpha^{2}+48 \alpha+24\right) \theta^{5} \\
+\left(6 \alpha^{4}+38 \alpha^{3}+84 \alpha^{2}+76 \alpha+24\right) \theta^{4}+\left(3 \alpha^{5}+29 \alpha^{4}+93 \alpha^{3}+121 \alpha^{2}+54 \alpha\right) \theta^{3} \\
+\left(\alpha^{6}+14 \alpha^{5}+57 \alpha^{4}+94 \alpha^{3}+62 \alpha^{2}+12 \alpha\right) \theta^{2}+\left(3 \alpha^{6}+21 \alpha^{5}+51 \alpha^{4}+51 \alpha^{3}+18 \alpha^{2}\right) \theta \\
+\left(2 \alpha^{6}+10 \alpha^{5}+18 \alpha^{4}+14 \alpha^{3}+4 \alpha^{2}\right)
\end{array}\right.}{\theta^{3}\left\{\theta^{2}+\alpha \theta+\alpha(\alpha+1)\right\}^{3}}
\end{aligned}
$$




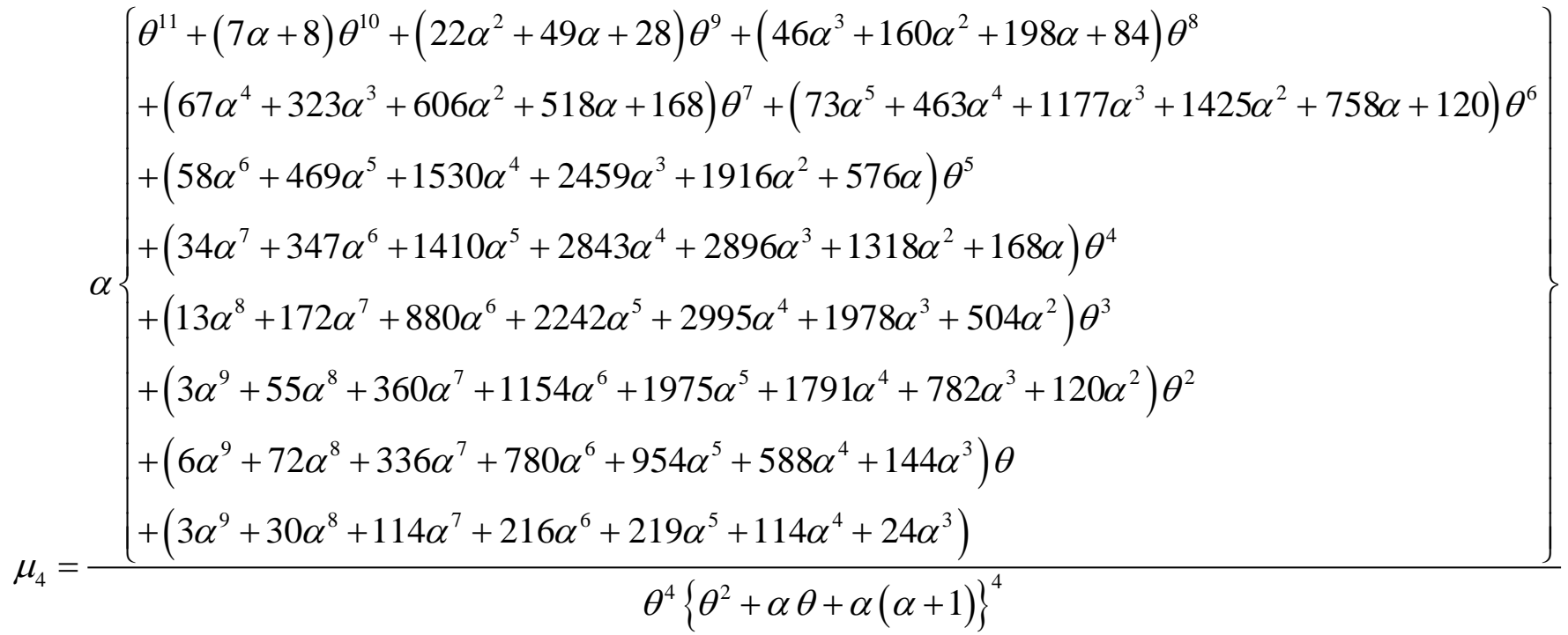

D. Coefficient of Variation, Skewness, kurtosis and Index of Dispersion

The coefficient of variation $(C . V)$, coefficient of Skewness $\left(\sqrt{\beta_{1}}\right)$, coefficient of Kurtosis $\left(\beta_{2}\right)$ and index of dispersion $(\gamma)$ of the P-WSD (2.2) are thus obtained as

$$
C . V=\frac{\sigma}{\mu_{1}^{\prime}}=\frac{\sqrt{\left\{\begin{array}{l}
\theta^{5}+2(\alpha+1) \theta^{4}+\left(3 \alpha^{2}+7 \alpha+4\right) \theta^{3}+14\left(2 \alpha^{3}+8 \alpha^{2}+12 \alpha+6\right) \theta^{2} \\
+\left(\alpha^{4}+6 \alpha^{3}+11 \alpha^{2}+6 \alpha\right) \theta+\left(\alpha^{4}+4 \alpha^{3}+5 \alpha^{2}+2 \alpha\right)
\end{array}\right\}}}{\sqrt{\alpha}\left\{\theta^{2}+(\alpha+1) \theta+(\alpha+1)(\alpha+2)\right\}}
$$

$\sqrt{\beta_{1}}=\frac{\mu_{3}}{\mu_{2}^{3 / 2}}=\frac{\left\{\begin{array}{l}\theta^{8}+(3 \alpha+4) \theta^{7}+\left(6 \alpha^{2}+16 \alpha+10\right) \theta^{6}+\left(7 \alpha^{3}+31 \alpha^{2}+48 \alpha+24\right) \theta^{5} \\ +\left(6 \alpha^{4}+38 \alpha^{3}+84 \alpha^{2}+76 \alpha+24\right) \theta^{4}+\left(3 \alpha^{5}+29 \alpha^{4}+93 \alpha^{3}+121 \alpha^{2}+54 \alpha\right) \theta^{3} \\ +\left(\alpha^{6}+14 \alpha^{5}+57 \alpha^{4}+94 \alpha^{3}+62 \alpha^{2}+12 \alpha\right) \theta^{2}+\left(3 \alpha^{6}+21 \alpha^{5}+51 \alpha^{4}+51 \alpha^{3}+18 \alpha^{2}\right) \theta \\ +\left(2 \alpha^{6}+10 \alpha^{5}+18 \alpha^{4}+14 \alpha^{3}+4 \alpha^{2}\right)\end{array}\right.}{\sqrt{\alpha}\left\{\begin{array}{l}\theta^{5}+2(\alpha+1) \theta^{4}+\left(3 \alpha^{2}+7 \alpha+4\right) \theta^{3}+14\left(2 \alpha^{3}+8 \alpha^{2}+12 \alpha+6\right) \theta^{2} \\ +\left(\alpha^{4}+6 \alpha^{3}+11 \alpha^{2}+6 \alpha\right) \theta+\left(\alpha^{4}+4 \alpha^{3}+5 \alpha^{2}+2 \alpha\right)\end{array}\right\}}$ 


$$
\begin{aligned}
& \left(\theta^{11}+(7 \alpha+8) \theta^{10}+\left(22 \alpha^{2}+49 \alpha+28\right) \theta^{9}+\left(46 \alpha^{3}+160 \alpha^{2}+198 \alpha+84\right) \theta^{8}\right. \\
& +\left(67 \alpha^{4}+323 \alpha^{3}+606 \alpha^{2}+518 \alpha+168\right) \theta^{7}+\left(73 \alpha^{5}+463 \alpha^{4}+1177 \alpha^{3}+1425 \alpha^{2}+758 \alpha+120\right) \theta^{6} \\
& +\left(58 \alpha^{6}+469 \alpha^{5}+1530 \alpha^{4}+2459 \alpha^{3}+1916 \alpha^{2}+576 \alpha\right) \theta^{5} \\
& \left\{+\left(34 \alpha^{7}+347 \alpha^{6}+1410 \alpha^{5}+2843 \alpha^{4}+2896 \alpha^{3}+1318 \alpha^{2}+168 \alpha\right) \theta^{4}\right. \\
& +\left(13 \alpha^{8}+172 \alpha^{7}+880 \alpha^{6}+2242 \alpha^{5}+2995 \alpha^{4}+1978 \alpha^{3}+504 \alpha^{2}\right) \theta^{3} \\
& +\left(3 \alpha^{9}+55 \alpha^{8}+360 \alpha^{7}+1154 \alpha^{6}+1975 \alpha^{5}+1791 \alpha^{4}+782 \alpha^{3}+120 \alpha^{2}\right) \theta^{2} \\
& +\left(6 \alpha^{9}+72 \alpha^{8}+336 \alpha^{7}+780 \alpha^{6}+954 \alpha^{5}+588 \alpha^{4}+144 \alpha^{3}\right) \theta \\
& \beta_{2}=\frac{\mu_{4}}{\mu_{2}^{2}}=\frac{\left(+\left(3 \alpha^{9}+30 \alpha^{8}+114 \alpha^{7}+216 \alpha^{6}+219 \alpha^{5}+114 \alpha^{4}+24 \alpha^{3}\right)\right.}{\alpha\left\{\begin{array}{l}
\theta^{5}+2(\alpha+1) \theta^{4}+\left(3 \alpha^{2}+7 \alpha+4\right) \theta^{3}+14\left(2 \alpha^{3}+8 \alpha^{2}+12 \alpha+6\right) \theta^{2} \\
+\left(\alpha^{4}+6 \alpha^{3}+11 \alpha^{2}+6 \alpha\right) \theta+\left(\alpha^{4}+4 \alpha^{3}+5 \alpha^{2}+2 \alpha\right)
\end{array}\right\}} \\
& \gamma=\frac{\sigma^{2}}{\mu_{1}^{\prime}}=\frac{\left\{\begin{array}{l}
\theta^{5}+2(\alpha+1) \theta^{4}+\left(3 \alpha^{2}+7 \alpha+4\right) \theta^{3}+14\left(2 \alpha^{3}+8 \alpha^{2}+12 \alpha+6\right) \theta^{2} \\
+\left(\alpha^{4}+6 \alpha^{3}+11 \alpha^{2}+6 \alpha\right) \theta+\left(\alpha^{4}+4 \alpha^{3}+5 \alpha^{2}+2 \alpha\right)
\end{array}\right\}}{\theta\left\{\theta^{2}+\alpha \theta+\alpha(\alpha+1)\right\}\left\{\theta^{2}+(\alpha+1) \theta+(\alpha+1)(\alpha+2)\right\}}
\end{aligned}
$$

Behaviors of coefficient of variation, coefficient of skewness, coefficient of kurtosis and index of dispersion of P-WSD for varying values of parameters $\theta$ and $\alpha$ have been shown graphically in figure 2 .
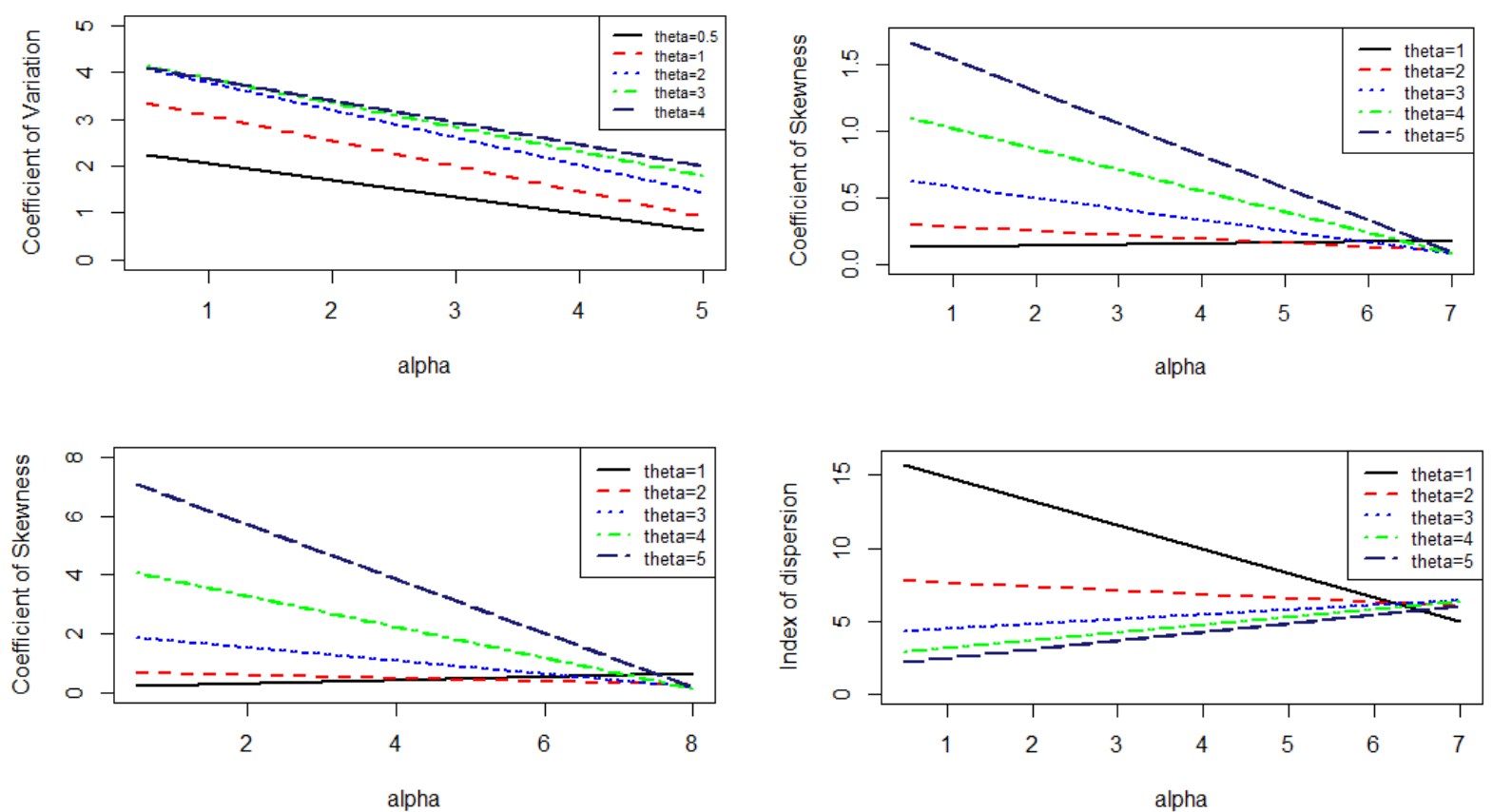

Fig. 2: Behaviors of coefficient of variation, coefficient of skewness, coefficient of kurtosis and index of dispersion of P-WSD for varying values of parameters $\theta$ and $\alpha$ 


\section{E. Increasing Hazard Rate and Unimodality}

We have

$$
\frac{P_{4}(x+1 ; \theta, \alpha)}{P_{4}(x ; \theta, \alpha)}=\left(\frac{x+\alpha}{x+1}\right)\left(\frac{1}{\theta+1}\right)\left[1+\frac{2 x+(\theta+2 \alpha+3)}{x^{2}+(\theta+2 \alpha+2) x+\left\{\theta^{2}+(\alpha+2) \theta+\left(\alpha^{2}+2 \alpha+1\right)\right\}}\right] .
$$

It can be easily verified that this is a decreasing function in $x$, and hence $P_{4}(x ; \theta, \alpha)$ is log-concave. Now using the results of relationship between log-concavity, unimodality and increasing hazard rate (IHR) of discrete distributions available in Grandell [20], it can concluded that P-WSD has an increasing hazard rate and is unimodal.

\section{MAXIMUM LIKELIHOOD ESTIMATION}

Let $\left(x_{1}, x_{2}, \ldots, x_{n}\right)$ be a random sample of size $n$ from the P-WSD (2.2) and let $f_{x}$ be the observed frequency in the sample corresponding to $X=x(x=1,2,3, \ldots, k)$ such that $\sum_{x=1}^{k} f_{x}=n$, where $k$ is the largest observed value having non-zero frequency. The log- likelihood function of P-WSD (2.2) can be given by

$$
\begin{aligned}
\log L= & n\left[(\alpha+2) \log \theta-(\alpha+2) \log (\theta+1)-\log \Gamma(\alpha)-\log \left\{\theta^{2}+\alpha \theta+\alpha(\alpha+1)\right\}\right] \\
& -\sum_{x=1}^{k} x f_{x} \log (\theta+1)+\sum_{x=1}^{k} f_{x}[\log \Gamma(x+\alpha)-\log (x+1)] \\
& +\sum_{x=1}^{k} f_{x} \log \left[x^{2}+(\theta+2 \alpha+2) x+\left\{\theta^{2}+(\alpha+2) \theta+\left(\alpha^{2}+2 \alpha+1\right)\right\}\right]
\end{aligned}
$$

The maximum likelihood estimates $(\hat{\theta}, \hat{\alpha})$ of parameters $(\theta, \alpha)$ of P-WSD (2.2) is the solutions of the following loglikelihood equations

$$
\begin{aligned}
& \frac{\partial \log L}{\partial \theta}=\frac{n(\alpha+2)}{\theta(\theta+1)}-\frac{n(2 \theta+\alpha)}{\theta^{2}+\alpha \theta+\alpha(\alpha+1)}-\frac{n \bar{x}}{\theta+1} \\
& +\sum_{x=1}^{k} \frac{(x+2 \theta+\alpha+2) f_{x}}{x^{2}+(\theta+2 \alpha+2) x+\left\{\theta^{2}+(\alpha+2) \theta+\left(\alpha^{2}+2 \alpha+1\right)\right\}}=0 \\
& \frac{\partial \log L}{\partial \alpha}=n \log \left(\frac{\theta}{\theta+1}\right)-n \psi(\alpha)-\frac{n(\theta+2 \alpha+1)}{\theta^{2}+\alpha \theta+\alpha(\alpha+1)}+\sum_{x=1}^{k} f_{x} \psi(x+\alpha) \\
& \quad+\sum_{x=1}^{k} \frac{\{\theta+2(x+\alpha+1)\} f_{x}}{x^{2}+(\theta+2 \alpha+2) x+\left\{\theta^{2}+(\alpha+2) \theta+\left(\alpha^{2}+2 \alpha+1\right)\right\}}=0
\end{aligned}
$$

where $\bar{x}$ is the sample mean and $\psi(x+\alpha)=\frac{d}{d \alpha} \log \Gamma(x+\alpha)$ and $\psi(\alpha)=\frac{d}{d \alpha} \log \Gamma(\alpha)$ are digamma functions. These two log- likelihood equations do not seem to be solved directly because they cannot be expressed in closed forms. But 
the MLE's $(\hat{\theta}, \hat{\alpha})$ of parameters $(\theta, \alpha)$ can be obtained directly by solving the log- likelihood equation using NewtonRaphson iteration method available in $\mathrm{R}-$ Software till sufficiently close estimates of $\hat{\theta}$ and $\hat{\alpha}$ are obtained. The initial values of parameters are taken as $\theta=0.5$ and $\alpha=0.5$.

\section{GOODNESS OF FIT}

In this section the applications of the P-WSD has been discussed with two count datasets from biological sciences. The dataset in table 1 is the data regarding the number of European red mites on apple leaves, available in Bliss [21]. The dataset in table 2 is the number of micronuclei after exposure at dose 4 Gy of $\gamma$ irradiation, counted using the cytochalasin B method and available in Piug and Valero [22]. The goodness of fit of P-WSD has been compared with the goodness of fit given by Poisson distribution (PD), PLD, PSD, and P-WLD. Note that the estimates of the parameters are based on maximum likelihood for all the considered distributions. Based on the values of chi-square $\left(\chi^{2}\right),-2 \log L$ and AIC (Akaike Information criterion), it is obvious that P-WSD is competing well with the considered distributions and gives better fit. Note that AIC has been calculated using the formula $A I C=-2 \log L+2 k$, where $k$ is the number of parameters involved in the distribution.

Table 1: Observed and Expected number of European red mites on Apple leaves, available in Bliss [21]

\begin{tabular}{|c|c|c|c|c|c|c|}
\hline $\begin{array}{c}\text { Number of } \\
\text { Red mites per } \\
\text { leaf }\end{array}$ & $\begin{array}{c}\text { Observed } \\
\text { frequency }\end{array}$ & \multicolumn{5}{|c|}{ Expected frequency } \\
\cline { 2 - 7 } & & PD & PLD & PSD & P-WLD & P-WSD \\
\hline 0 & 70 & 47.6 & 67.2 & 66.4 & 69.8 & 70.1 \\
1 & 38 & 54.6 & 38.9 & 39.3 & 36.8 & 36.1 \\
2 & 17 & 31.3 & 21.2 & 21.8 & 20.1 & 20.3 \\
3 & 10 & 11.9 & 11.1 & 11.5 & 10.9 & 11.2 \\
4 & 9 & 3.4 & 5.7 & 5.7 & 5.8 & 6.0 \\
5 & 3 & 0.8 & 2.8 & 2.8 & 3.0 & 3.1 \\
6 & 2 & 0.2 & 1.4 & 1.3 & 1.6 & 1.6 \\
7 & 1 & 0.1 & 0.9 & 0.6 & 0.8 & 0.8 \\
8 & 0 & 0.1 & 0.8 & 0.6 & 1.2 & 0.8 \\
\hline Total & 150 & 150.0 & 150.0 & 150.0 & 150.0 & 150.0 \\
\hline ML estimates & & $\hat{\theta}=1.14666 \hat{\theta}=1.26010$ & $\hat{\theta}=1.65330$ & $\hat{\theta}=1.09141$ & $\hat{\theta}=1.37885$ \\
& & & & & $\hat{\alpha}=0.82194$ & $\hat{\alpha}=0.73252$ \\
\hline Standard & & 0.08743 & 0.11390 & 0.12331 & 0.26231 & 0.27269 \\
Errors & & & & & 0.25230 & 0.23840 \\
& & & & & & \\
\hline$\chi^{2}$ & & 26.50 & 2.49 & 3.49 & 2.41 & 2.27 \\
\hline d.f & & 2 & 4 & 4 & & 3 \\
\hline p-value & & 0.0000 & 0.5595 & 0.4794 & 0.4917 & 0.51829 \\
\hline$-2 \log L$ & & 485.61 & 445.02 & 445.27 & 425.35 & 425.13 \\
\hline AIC & & 487.61 & 447.02 & 447.27 & 429.35 & 429.13 \\
\hline
\end{tabular}


Table 2: Number of micronuclei after exposure at dose 4 Gy of $\gamma$ irradiation, counted using the cytochalasin B method and available in Piug and Valero [22]

\begin{tabular}{|c|c|c|c|c|c|c|}
\hline \multirow{2}{*}{$\begin{array}{l}\text { Number of } \\
\text { micronuclei }\end{array}$} & \multirow{2}{*}{$\begin{array}{l}\text { Observed } \\
\text { frequency }\end{array}$} & \multicolumn{5}{|c|}{ Expected frequency } \\
\hline & & $\mathrm{PD}$ & PLD & PSD & P-WLD & P-WSD \\
\hline 0 & 1974 & 1816.0 & 2396.8 & 2360.5 & 1966.0 & 1966.4 \\
\hline 1 & 1674 & 1839.9 & 1300.3 & 1311.2 & 1695.5 & 1694.7 \\
\hline 2 & 869 & 932.1 & 668.8 & 687.2 & 857.6 & 857.8 \\
\hline 3 & 342 & 314.8 & 332.1 & 342.5 & 331.7 & 331.9 \\
\hline 4 & 102 & 79.7 & 160.9 & 163.9 & 108.5 & 108.5 \\
\hline 5 & 26 & 16.1 & 76.5 & 75.9 & 31.5 & 31.5 \\
\hline 6 & 13 & 2.7 & 35.8 & 34.2 & 8.4 & 8.4 \\
\hline 7 & 2 & 1.6 & 30.8 & 26.6 & 2.8 & 2.8 \\
\hline Total & 5002 & 5002.0 & 5002.0 & 5002.0 & 5002.0 & 5002.0 \\
\hline ML estimates & & $\hat{\theta}=1.01319$ & $\hat{\theta}=1.38736$ & $\hat{\theta}=1.77716$ & $\begin{array}{l}\hat{\theta}=5.97458 \\
\hat{\alpha}=5.57089\end{array}$ & $\begin{array}{l}\hat{\theta}=6.34851 \\
\hat{\alpha}=5.47608\end{array}$ \\
\hline $\begin{array}{c}\text { Standard } \\
\text { Errors }\end{array}$ & & 0.01423 & 0.02251 & 0.02378 & $\begin{array}{l}0.83470 \\
0.83790 \\
\end{array}$ & $\begin{array}{l}0.83783 \\
0.83658 \\
\end{array}$ \\
\hline$\chi^{2}$ & & 62.21 & 337.08 & 302.5 & 3.41 & 3.37 \\
\hline d.f & & 4 & 5 & 5 & 4 & 4 \\
\hline p-value & & 0.0000 & 0.0000 & 0.0000 & 0.4909 & 0.4979 \\
\hline$-2 \log L$ & & 13535.82 & 13836.70 & 13799.20 & 15597.78 & 15597.75 \\
\hline AIC & & 13537.82 & 13836.70 & 13801.20 & 15601.78 & 15601.75 \\
\hline
\end{tabular}

The fitted plots of the considered distributions for datasets in table 1 and 2 have been shown in figure 3. It is obvious that P-WSD gives much closer fit over other mixed Poisson distributions.
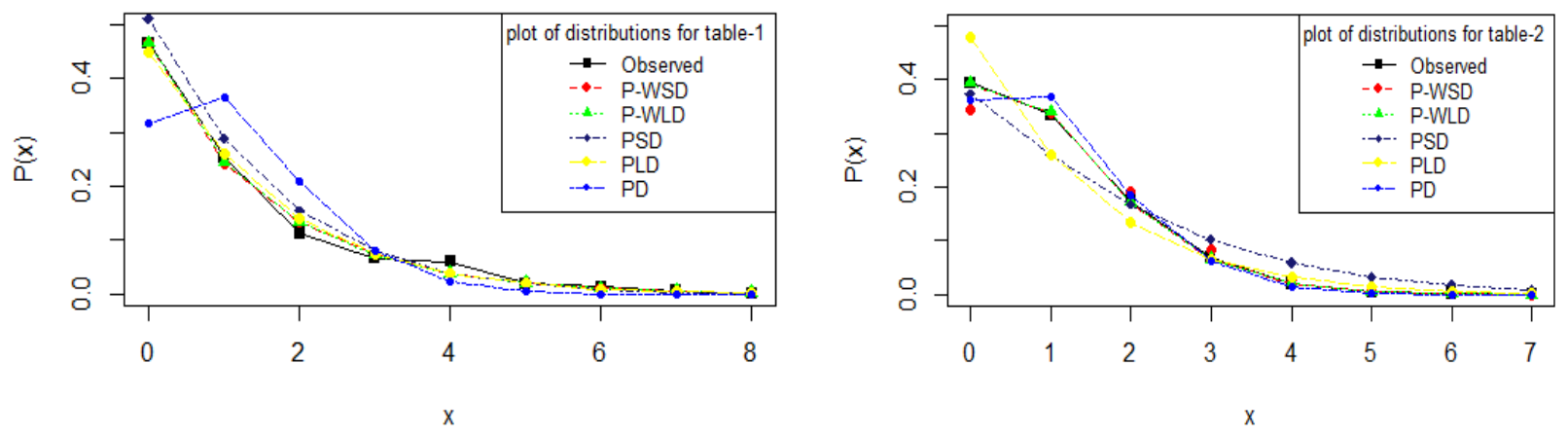

Fig.3. Fitted probability plots for considered discrete distributions

\section{VI.CONCLUDING REMARKS}

The Poisson-weighted Sujatha distribution (P-WSD) which includes Poisson-Sujatha distribution (PSD) as particular case has been proposed by compounding Poisson distribution with a two-parameter weighted Sujatha distribution. Its statistical properties including moments, coefficient of variation, skewness, kurtosis, index of dispersion, unimodality and increasing 
hazard rate have been discussed. Maximum likelihood estimation has been explained for estimating its parameters. Applications of the distribution have been discussed with two count datasets from biological sciences and its goodness of fit has been compared with other discrete distributions having over-dispersion. Since the fit by P-WSD has been found quite satisfactory over, PD, PLD, PSD, and P-WLD, P-WSD can be considered an important Poisson mixed distribution in distribution theory.

\section{REFERENCES}

[1] M. Raghavachari,, A.,Srinivasam, and P., Sullo, "Poisson mixture yield models for integrated circuits - A critical review", Microelectronics Reliability, 37 (4), $565-580,1997$

[2] D. Karlis and E., Xekalaki , “ Mixed Poisson distributions”, International Statistical review, 73(1), 35 - 58,2005

[3] H.H., Panjeer, "Mixed Poisson distributions. In Encyclopedia of Actuarial Science”, John Wiley and Sons Ltd, Hoboken, New Jersey, USA., 2006

[4] M., Greenwood, M. and G.U., Yule, "An inquiry into the nature of frequency distributions representative of multiple happenings with particular reference to the multiple attacks of disease or of repeated accidents", Journal of the Royal Statistical Society, 83(2), 115 - 121, 1920

[5] M., Sankaran , “ The discrete Poisson-Lindley distribution”, Biometrics, 26, 145- 149,1970

[6] D.V., Lindley, "Fiducial distributions and Bayes' Theorem", Journal of the Royal Statistical Society, Series B, 20, 102 - 107, 1958

[7] R., Shanker, "The Discrete Poisson-Akash Distribution", International Journal of Probability and Statistics, 6(1), 1 -10,2017

[8] R., Shanker, “Akash distribution and Its Applications,” International Journal of Probability and Statistics, 4(3), 65 -75, 2015

[9] E., Mahmoudi and H., Zakerzadeh , "Generalized Poisson-Lindley distribution” Communication in Statistics-Theory and Methods, 39, 1785 1798,2010

[10] H., Zakerzadeh and A. Dolati , "Generalized Lindley distribution”, Journal of Mathematical extension, 3 (2), 13 - 25, 2009

[11] H., Zamani, N., Ismail and P., Faroughi, "Poisson- Weighted exponential univariate version and regression model with applications", Journal of Mathematics and Statistics, 10(2), 148 - 154, 2014

[12] H., Zamani and Ismail, "Negative binomial-Lindley distribution and its Applications", Journal of Mathematics and Statistics, 6(1), 4 - 9, 2010

[13] M.M.E., Abd El-Monsef and N.M., Sohsah , “ Poisson-Weighted Lindley Distribution”, Jokull Journal, 64(5), 192 - 202, 2014

[14] M.E., Ghitany ,F., Alqallaf , D.K., Al-Mutairi and H.A., Husain, "A two-parameter weighted Lindley distribution and its applications to survival data", Mathematics and Computers in simulation, 81, 1190-1201,2011

[15] R., Shanker (2016 b): The Discrete Poisson-Sujatha Distribution, International Journal of $\quad$ Probability and Statistics, $5(1), 1$ - 9.

[16] R., Shanker (2016 a): Sujatha Distribution and Its Applications, Statistics in Transition-New series, 17(3), 1- 20.

[17] R., Shanker and K.K.., Shukla (2018): A two-parameter Weighted Sujatha distribution and Its Application to Model Lifetime data, International Journal of Applied Mathematics and Statistics, 57 (3), 106 - 121

[18] R., Shanker, K.K., Shukla and F., Hagos (2016): On Weighted Lindley distribution and Its Applications to Model Lifetime Data, Jacobs Journal of Biostatistics, 1(1), 1- 9

[19] R., Shanker, K.K., Shukla and A., Mishra, (2017): A Three- parameter weighted Lindley distribution and its applications to model survival time data, Statistics in Transition-New Series, 18 (2), 291 - 300.

[20] J., Grandell (1997): Mixed Poisson Processes, Chapman\& Hall, London.

[21] C.I., Bliss (1953): Fitting negative binomial distribution to biological data, Biometrics, 9, 177 - 200.

[22] P., Piug and J. Valero (2006): Count data distribution- Some characterizations with applications, Journal of the American Statistical Association, 101 (473), $332-340$ '

\section{Authors Profile}

Prof. Rama Shanker has completed Ph.D in Statistics from Department of Statistics, Patna University, Patna, India. He has twenty one years of teaching and research experience and published 130 research papers in distribution theory in reputed journals of Statistics, Mathematics, and Biostatistics. He is the founding head of Department of Statistics at Eritrea Institute of Technology (EIT), Eritrea and founding Editor-in-Chief of 'Eritrean Journal of Science and Engineering (EJSE)'. He is the international advisory Board members of journals published from India, Singapore, Turkey and USA and reviewers of research papers in distribution theory. He has been working as international advisory committee members of conferences/seminars in Statistics and delivered several invited/plenary talks in Statistics and Statistics related disciplines.

Dr. Kamlesh Kumar Shukla is presently working as Associate Professor at Department of Statistics EIT, Eritrea since February, 2016. He has been awarded Ph.D. in Statistics from Banaras Hindu University, Varanasi, India, and Masters Degree in Statistics (Gold Medalist) in 1997.Dr. Shukla has worked as faculty member in the different International and National organizations and having more than 14 years of teaching experience. His areas of research are Distribution theory, Migration, Demography and Human Values. He has published more than 60 research papers in International and National Journals.

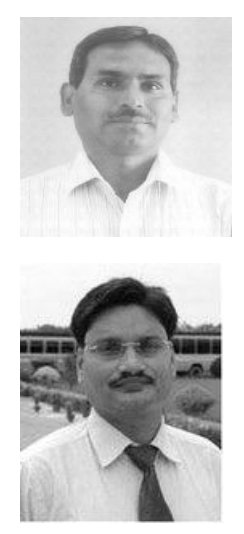

